\title{
Research on Damping Characteristics of Magneto-rheological Damper Used in Vehicle Seat Suspension
}

\author{
Farong Kou, Qinyu Sun,Pan Liu \\ College of Mechanical Engineering, Xi'an University of Science and \\ Technology, Xi'an,710054, China
}

\begin{abstract}
Based on the analysis of working principle and simplified model, a kind of magneto-rheological damper is designed and developed, which is used in vehicle seat suspension. Under different control currents and different excitation frequency inputs, damping characteristics experiments of magnetorheological damper are completed. The results indicate that the magnetorheological damper has good effectiveness for energy dissipation. The damping force increases with the improving of control current and excitation frequency and tends to balance.

Keywords: seat suspension, magneto-rheological damper, control current, damping characteristics, experiment.

\section{Introduction}

The magneto-rheological phenomenon for magneto-rheological fluid was found by Rabinow in 1940s. Under the external magnetic field, magneto-rheological fluid becomes from free-flowing liquid into the semi-solid in an instant, which has controllable yield strength. And this change is reversible[1].

Magneto-rheological damper with magneto-rheological fluid is a kind of semi-active damping controlled device. Its damping force is subject to controlled electric current. Magneto-rheological damper has some advantages, such as simple structure, small size, fast response, wide dynamic range, low energy consumption, good durability, and so on. Magneto-rheological damping control has not only the reliability of passive system but also strong adaptability of active control system, which is close to active control system through a certain control strategy.

This paper applies magneto-rheological damper to vehicle seat suspension. Based on the analysis of working principle and simplified model, a kind of magneto-rheological damper is designed and developed. Under a certain control currents and excitation frequency input, damping characteristics experiments of magneto-rheological damper are completed.
\end{abstract}




\section{The working principle of magneto-rheological damper}

\subsection{The basic structure of magneto-rheological damper}

Magneto-rheological damper includes the magneto-rheological fluid, cylinder block, piston,orifice,coil and other components[2]. The orifices are located on the piston. And coil windings, which can produce strong magnetic field, are installed around the orifice, as shown in Fig.1.

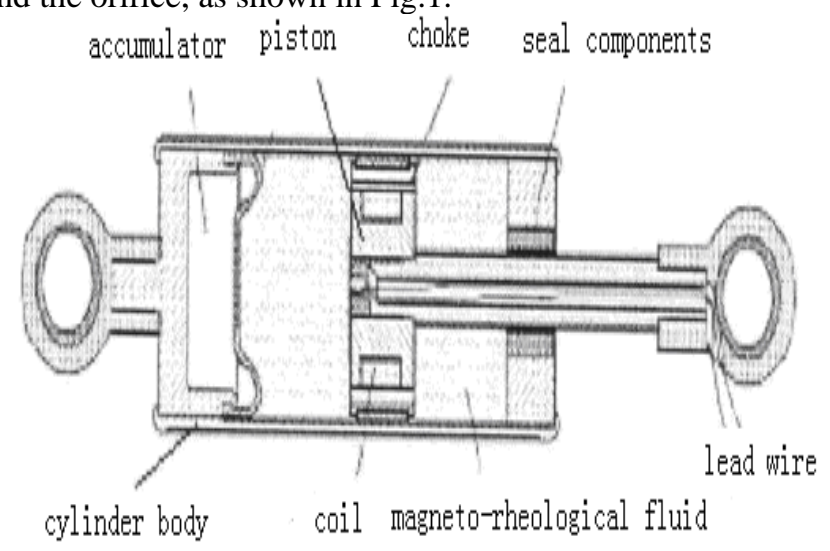

Fig.1: The structure of magneto-rheological damper

When the magneto-rheological damper installed in the seat suspension begin to work, the piston of magneto-rheological damper keeps reciprocating linear movement inside its cylinder. According to position feedback signal of damper piston, the controller sends out specific drive-control command under a certain control law. Under strong magnetic field, ferromagnetic particles in the magnetorheological fluid form a "chain" between the orifice, which changes the surface viscosity of magneto-rheological fluid and controls the flow characteristics of magneto-rheological fluid in the damping throttling channel. It changes the pressure difference between left and right working cavity for damper piston. As a result, the damping force magneto-rheological damper is adjusted[3], as shown in Fig.2.

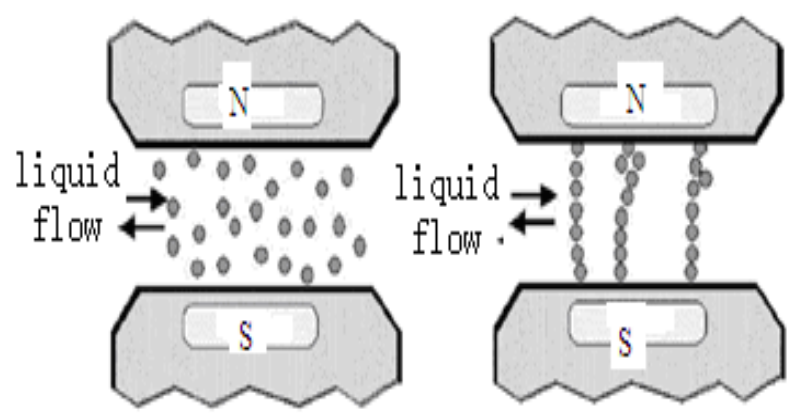

Fig.2: The flow characteristics of magneto-rheological liquid 


\subsection{Simplified model of magneto-rheological damper}

Ignoring the influence of damper friction and fluid inertia, and assuming that magneto-rheological fluid of magneto-rheological damper is incompressible [46] , the upper and lower working chamber both work in the uniform pressure, regarding the damping force of magneto-rheological damper as work flow mode damping force and shear mode damping force stack together, under the mixed mode, the expression of calculating damping force for magneto-rheological damper can be derived as:

$$
F=-\left(\frac{24 \eta A_{p}^{2} l}{b h^{3}}+\frac{2 \eta b l}{h}\right) \nu_{0}-\left(\frac{4 l A_{P}}{\eta}+2 b l\right) \tau_{y} .
$$

In Eq. $1, \eta$ represents the dynamic viscosity of fluid, $l$ represents the length of the work plates, $h$ represents the distance of the work plates, $v_{0}$ represents the moving speed of piston pole, $\tau_{y}$ represents the shear stress of magnetorheological fluid, $A_{p}$ represents the effective area of piston, $b$ represents the width of the work plates.

As seen in Eq. 1, the damping force of magneto-rheological damper is equal to the sum of viscous damping and coulomb damping forces. Viscous damping force can be derived by piston velocity through a function relation; Coulomb damping force can be derived by magneto-rheological fluid yield stress through a function relation, magneto-rheological fluid yield stress is controlled by the magnetic field strength, also has a function with control current, therefore, Eq. 1 can be turned into:

$$
F=-C_{e} v_{0}+F_{M R} \cdot
$$

In Eq.2, $C_{e}$ represents the equivalent viscous damping coefficient, $F_{M R}$ represents the coulomb damping force. Through experimental study of shear stress and shear strain rate of magneto-rheological fluid and unitary nonlinear regression fitting function, ultimately identify the function of the magneto-rheological fluid yield stress and the magnetic induction, it can be expressed as:

$$
\begin{gathered}
\tau_{y}=1.43 \times 10^{-6} B^{2}+4.743 \times 10^{-3} B-1 \\
B=\mu N_{c} I / 2 h
\end{gathered}
$$

In Eq.3 and Eq.4, $h$ represents the damping channel gap, $\mu$ represents the magneto-rheological fluid permeability, $B$ represents the magnetic induction of the damping channel, $N_{c}$ represents the coil turns, $I$ represents the coil excitation control current.

Joint Eq.1, Eq.2 and Eq.3, a formula for calculating damping force of magneto-rheological damper under certain control current is concluded:

$$
F=-C_{e} v_{0}+\left(a_{1} I^{2}+a_{2} I+a_{3}\right) \operatorname{sgn}\left(v_{0}\right)
$$

Through the experiment and calculation, which used in this study for calculating damping force of magneto-rheological damper is: 


$$
F=-180 v_{0}+\left(0.086 I^{2}+0.38 I+110\right) \operatorname{sgn}\left(v_{0}\right)
$$

\section{Damping characteristics of magneto-rheological damper 3.1 The experimental system of vehicle seat suspension using magneto- rheological damper}

This article applies magneto-rheological damper to semi-active control of seat suspension, under the certain piston speed, by controlling the current in the coil to generate the corresponding magnetic field intensity, thereby forming certain damping force. Major equipments for the experiment of the damping characteristics of magneto-rheological damper include sample of magnetorheological damper, the MTS testing machine, and data acquisition system[7-8]. Magneto-rheological damper uses Tider-MRD-50-6 which is in the production of Technology Co., Ltd. Harbin Tai Da Er, the outer diameter of cylinder reaches $45 \mathrm{~mm}$, stroke reaches $\pm 15 \mathrm{~mm}$, power reaches $4 \mathrm{~W}$, maximum current reaches $2 \mathrm{~A}$. Test the indicator characteristics of magneto-rheological damper on the MTS testing machine, the test site as shown in Fig.3and Fig.4.

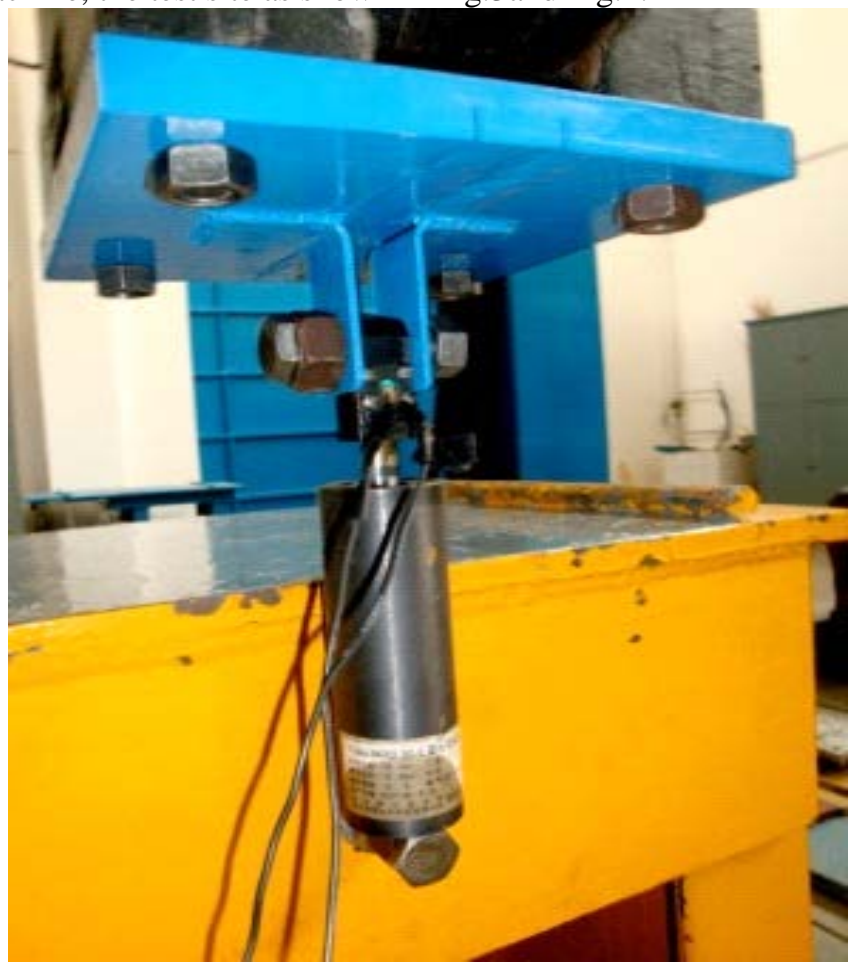

Fig.3: Magneto-rheological damper 


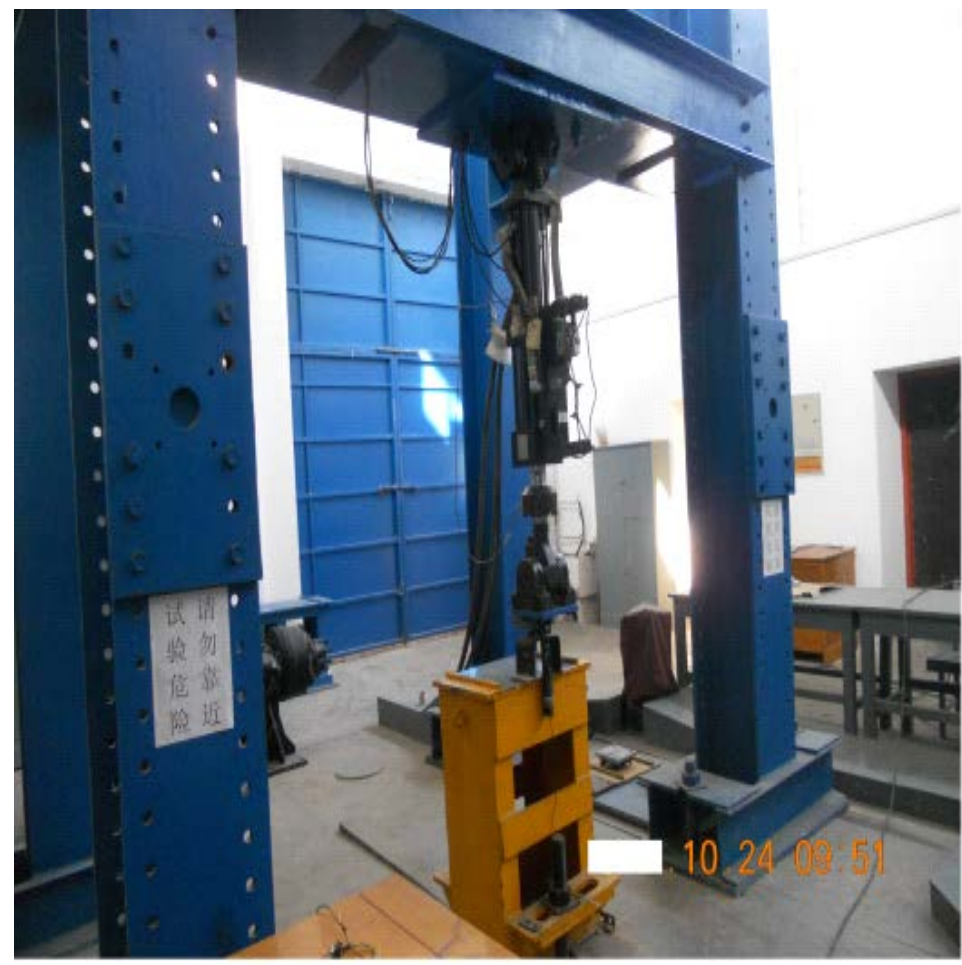

Fig.4: Test system

In the experiments, the vibration excitation of hydraulic cylinder makes the magneto-rheological damper piston of seat suspension within the cylinder for reciprocating motion, force sensor and displacement sensor tests damping force and the vibration displacement of hydraulic cylinder, this displacement is the relative displacement of the magneto-rheological damper piston.

\subsection{Experimental study of magneto-rheological damper used In vehicle seat suspension}

Through bench test, the relation curve of the damping force and piston displacement, for magneto-rheological damper used in vehicle seat suspension, was got in Fig.5and Fig.6. 


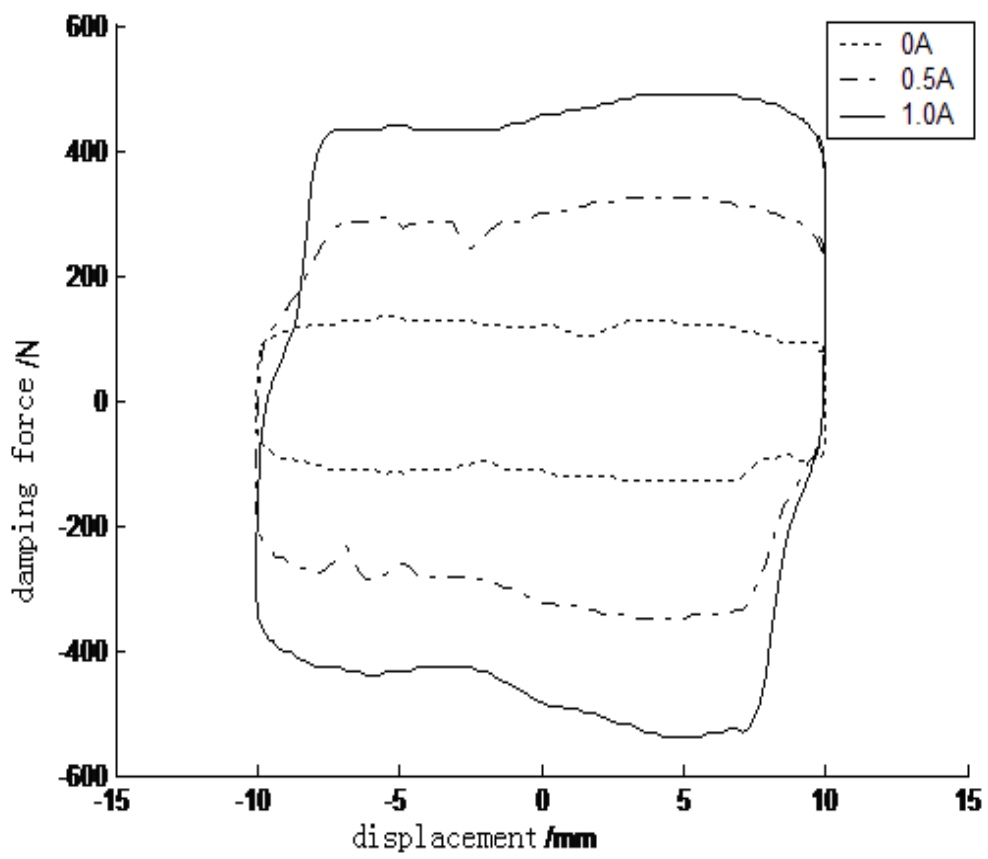

Fig.5: Damping force vs. displacement under different current

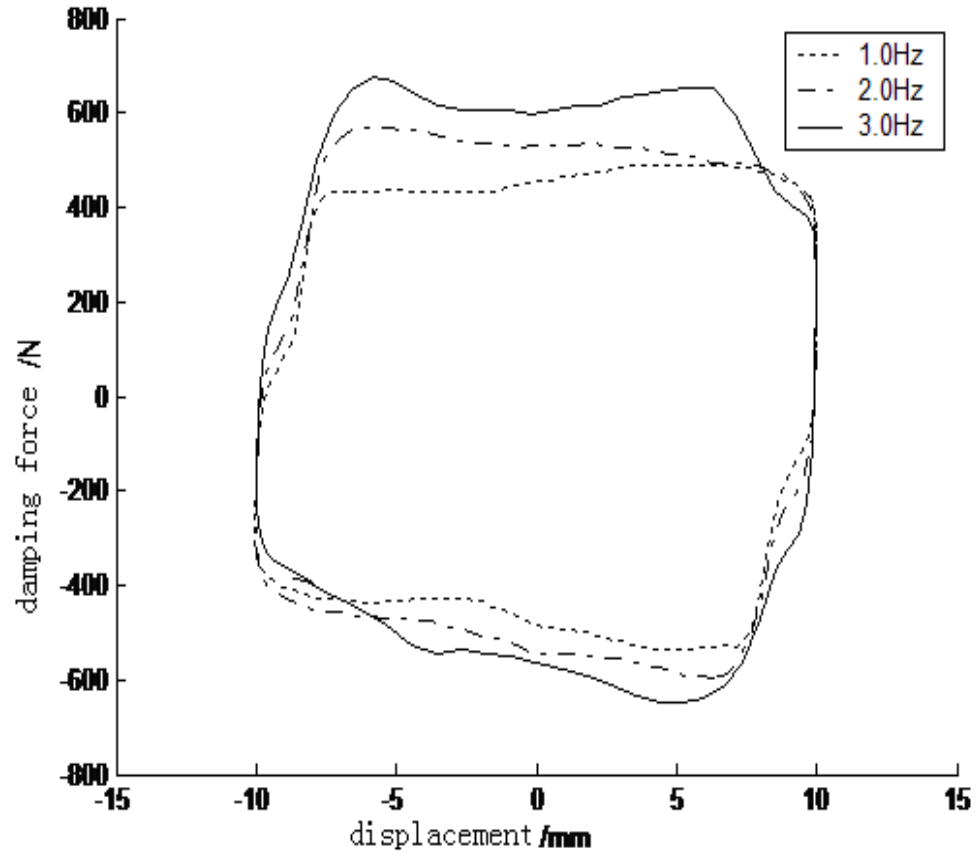

Fig.6: Damping force vs. displacement under different frequency 
By comparison and analysis of experimental results, can get the following conclusions[9]:

i) The curve relationship of the damping force and piston displacement of magneto-rheological damper is full, it shows that the effect of vibration attenuation and energy dissipation of magneto-rheological damper is good.

ii) Under the sine excitation input whose excitation amplitude is $10 \mathrm{~mm}$ and excitation frequency is $1 \mathrm{~Hz}$, the damping force of magneto-rheological damper increases along with the rising of the control current (0A to $1 \mathrm{~A})$.

iii) For the same control current (such as $1.0 \mathrm{~A}$ ), the damping force values of magneto-rheological damper remains constant. when the control current is $0 \mathrm{~A}$, damping force reaches the minimum; When the control current is $1 \mathrm{~A}$, damping force reaches the maximum.

iv) Under the input that the sine excitation amplitude is $10 \mathrm{~mm}$ and control current is $1.0 \mathrm{~A}$, the damping force of magneto-rheological damp increases along with the rising of the excitation frequency $(1.0 \mathrm{~Hz}$ to $3.0 \mathrm{~Hz})$, but increases modestly.

v) Along with the excitation frequency on the magneto-rheological damper and control current increases, the envelope area of the damping force - the piston displacement curve increases, the attenuation of the magneto-rheological damper and energy dissipation capacity also increases accordingly.

\section{Acknowledgements}

This work was financially supported by Scientific Research Program Funded by Shaanxi Provincial Education Department (Program No. 2013JK1008), The Project Supported by Natural Science Basic Research Plan in Shaanxi Province of China (Program No. 2014JM7271), Postdoctoral Science Foundation of China (Program No.2014M552553XB), National Natural Science Foundation of China (Program No.51275403), Doctoral Fund of Ministry of Education of China (Program No. 20126121120003), Postdoctoral initial funding of Xi'an University of Science and Technology (Program No.2013QDJ034).

\section{References}

[1] Yun Zhou, Ping Tan. Magneto-rheological damping control theory and technology.Bei jing: Science press,2007(In Chinese).

[2] Jie-ping Chen, Wu-wei Chen, Hui Zhu,et al. Design and test of vehicle single outstretch pole MRD.Transactions of the Chinese Society for Agricultural Machinery, 40(3), pp.5-10. 2009.

[3] S. J. MCMANUS, K. A. ST. CLAIR.Evaluation of vibration and shock attenuation performance of a suspension seat with a semi-active magnetorheological fluid damper. Journal of Sound and Vibration, 253(1), pp.313327,2002. 
[4] Zi-fan Fang, Zhao-xiang Deng. Study on simplified mechanical model of magneto-rheological damper. Engineering Mechanics,24(11), pp.32-35, 2007(In Chinese).

[5] Seung-Bok Choi,Young-Min Han.Vibration control of electrorheological seat suspension with human-body model using sliding mode control. Journal of Sound and Vibration, 303, pp.391-404,2007.

[6] Jian Zhang, Jin-qiu Zhang, Yun-guang Qi, et al.Modelling and experimental on MRF damper force of tracked vehicle. Noise and Vibration Control,31(2), pp.103-106,2011(In Chinese).

[7] Li-hong Shu, Yu-fei Wang. Experimental study on the performance of MR damper. Noise and Vibration Control, 30(3), pp.5-8,2010(In Chinese).

[8] Wan-jun Wang, Wei-gong Zhang, En-rong Wang. Experimental research on properties of magneto-rheological damper used in vehicle suspension. Automobile Technology, 42(9), pp.48-51,2009(In Chinese).

[9]Fa-rong Kou. Test on fuzzy control for vehicle active suspension with EHA[J]. Journal of Xi'an University of Science and Technology, 29(5), pp.589-593,2009(In Chinese). 